International Journal of Engineering \& Technology, 7 (2.7) (2018) 104-107
International Journal of Engineering \& Technology
SPC
Website: www.sciencepubco.com/index.php/IJET
Research Paper

\title{
Connecting vehicles using Li-Fi technology for reducing accidents
}

\author{
K.Raghava Rao ${ }^{1 *}$, K.Sai Sasi Preetham ${ }^{2}$, K.Edukondalu ${ }^{3}$ L.V.Ragavendra $\operatorname{Reddy}^{4}$ \\ ${ }^{I}$ Dept of Electronics and Computer Science Engineering, KLEF, Vaddeswaram. \\ ${ }^{2}$ Dept of Electronics and Computer Science Engineering, KLEF, Vaddeswaram. \\ ${ }^{3}$ Dept Of Electronics and Computer Science Engineering, KLEF, Vaddeswaram. \\ ${ }^{4}$ Dept of Electronics and Computer Science Engineering, KLEF, Vaddeswaram \\ *Email: saisasi888@gmail.com
}

\begin{abstract}
Li-FI technology is transferring of data using Led's in visible light spectrum. Basically Li-Fi is similar to that of Wi-Fi but this Li-Fi is 10 times faster than $\mathrm{Wi}-\mathrm{Fi}$, as Wi-Fi uses radio frequency which has some transmission limitation Li-Fi is very useful to replace such limitations. We can use this technology in many areas like hospitals air traffic control, vehicles and under water communications.in this Li-Fi technology we mainly use led's as a transmitter which will send stream of data by toggling with a speed that can't be identified by the human eye and photodiode as a receiver which converts light energy to electrical energy. Connecting vehicles can be obtained by replacing head and tail lamps of the vehicle with led's and photodiode should be attached at both front and rear end of the vehicle go get the data from both leading and following vehicles. By transferring the data about the motion of vehicle through led's we can reduce the collision of vehicles. This technology is also used to regulate the information about the traffic by replacing the street lights with led and transfer data about the traffic in that route for every vehicle that passes through.
\end{abstract}

Keywords: Light Emitting Diode(LED), Photodiode, Reduction of accidents, Connecting vehicles, Visible Light Communication

\section{Introduction}

\subsection{Light-Fidelity:}

$\mathrm{Li}-\mathrm{Fi}$ is same as that of Wi-Fi which is two directional. The inventor of this Li-Fi technology was Harald Haas and it is a type of visible light communication and a part of optical wireless communications(OWC) and also a supplement to Radio Frequency communication (Wi-Fi or cell organizes), or also a substitution in settings of information broadcasting. In future it will be the substitute

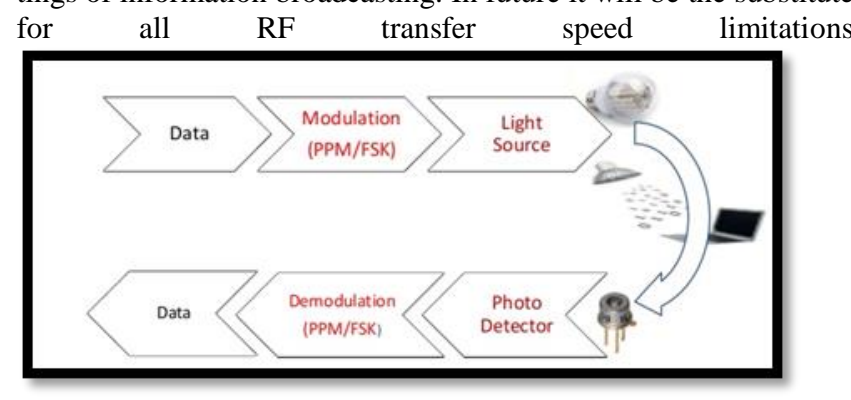

Fig:1 Block diagram of Li-Fi technology

Visible light communications(VLC) works by toggling Led's off and on with a range of Nano seconds which is too speedy to be identified by the human eye. As the li-fi led's might be kept directly to transmit records, they may be darkened to beneath human visibility even as as yet generating sufficient mild to convey information
Rather than radio frequency waves utilized by wi-fi, lights can't pass thru partitions and entryways. This makes it greater secure and makes it less worrying to manipulate who can accomplice with your machine. For something length of time that sincere substances like home windows are secured, access to a li-fi channel can be restricted to devices inside the room.

The gadget requires a transmitter and a receiver in each automobile in each rear and the front factors of the car. Because of this greater scenario can be applicable. In this, we plan model which depends on $\mathrm{Li}-\mathrm{Fi}$ innovation for vehicle to vehicle information transmission. Vehicle to vehicle correspondence is the best arrangement that has been utilized as a part of request to reduce vehicles accidents. In LI-Fi innovation information transmission through light for this reason wellspring of light is utilized as LED and the recipient will be a photograph diode. Vehicle to vehicle interchanges, for example, is one of the past patterns, which is a standout amongst the best systems that will actualize in cars to give security and a convention of correspondence. Enormous measure of research takes a shot at vehicle.

\subsection{Photo diode}

A photodiode is a semiconductor device that takes light as an input and produces electrical current as an output. The current will be generated when photons from the light falls on the photodiode. A minute electric is produced when light is absent. Photodiodes consists of optical filters, built-in lenses, and may have various surface areas. Surface area of a photo diode is inversely proportional to the response time. The common example in our daily life 
is solar panel that generates electricity for sun with a large surface area. Photodiodes are same that of regular semiconductor diodes the only change is That they'll be both uncovered (to locate vacuum uv or x-rays) or packaged with a window or optical fiber which permits light to reach the touchy a part of the device Pin junction is instead desired for photodiode instead of a $p-n$ junction, to growth the speed of reaction. A photodiode is designed to perform in opposite bias. The material used to make a photodiode is important to define its houses, due to the fact handiest photons with sufficient power to excite electrons throughout the fabric's bandgap will produce tremendous photocurrents.

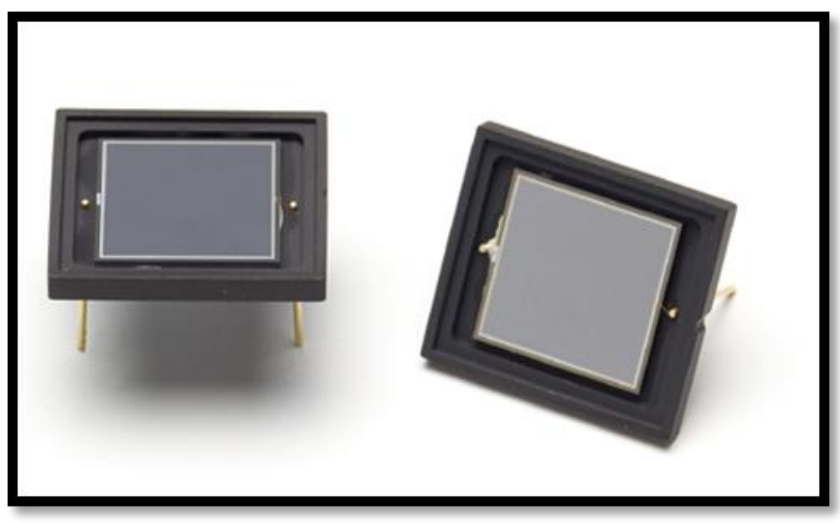

Fig:2 Photodiode

Table 1.1 Ranges of a photodiode

\begin{tabular}{|l|l|}
\hline Material & $\begin{array}{l}\text { Electromagnetic spec- } \\
\text { trum wavelength range } \\
\text { (nm) }\end{array}$ \\
\hline Silicon & 190 to1100 \\
\hline Germanium & 400 to1700 \\
\hline Indium gallium arsenide & 800 to2600 \\
\hline Lead(ii) supplied & $<1000$ to 3500 \\
\hline Mercury cadmium telluride & 400 to 1400 \\
\hline
\end{tabular}

\section{Principle pf operation:}

A photodiode is a semiconductor of PIN type or P-N type. when a photon of 0's energy moves towards the diode and creates an electron-gap combine. This is also known as the inward photoelectric impact. At the off risk that the retention takes place in the combination of exhaustion location, or one dispersion duration a long way from it, those transporters are taken off from the junction with the aid of the implicit electric powered area of the intake locale. Alongside these lines holes are moved towards the anode, and electrons towards the cathode, resulting the formation of photocurrent. The combination present day thru the photodiode is the whole thing of the dim (current that is created in the absence of light) and the photocurrent, so the dull modern-day ought to be limited to expand the affectability of the system.

\section{Photovoltaic mode}

At the factor whilst applied as a part of 0's inclination or photovoltaic mode, the circulation of photocurrent from the device is limited and a voltage created. This mode abuses the photovoltaic impact, that's the reason for solar based totally cells - a normal sun orientated mobile is only an intensive location photodiode.

\section{Photoconductive mode}

In this mode the diode is every now and again exchange uneven (with the cathode driven uncommon concerning the anode). This reduces the response time in light of the way that the additional rearrange inclination assembles the width of the admission layer, which decreases the convergence's capacitance. The exchange slant besides extends the senseless present day without loads substitute in the photocurrent. For a given unearthly scattering, the photocurrent is straightly with respect to the illuminance (and to the irradiance).

In spite of reality that this mode is speedier, the photoconductive mode tends to show more prominent electronic bustle. The spillage contemporary of a conventional stick diode is so low $(<1 \mathrm{na})$ that the Johnson- Nyquist racket of the store assurance in a typical circuit consistently runs the show.

\subsection{Led array}

Led arrays are assemblies of led bulbs that may be built the usage of several methods. Every method hinges at the manner and extent to which the chips themselves are packaged by using the led semiconductor

er.

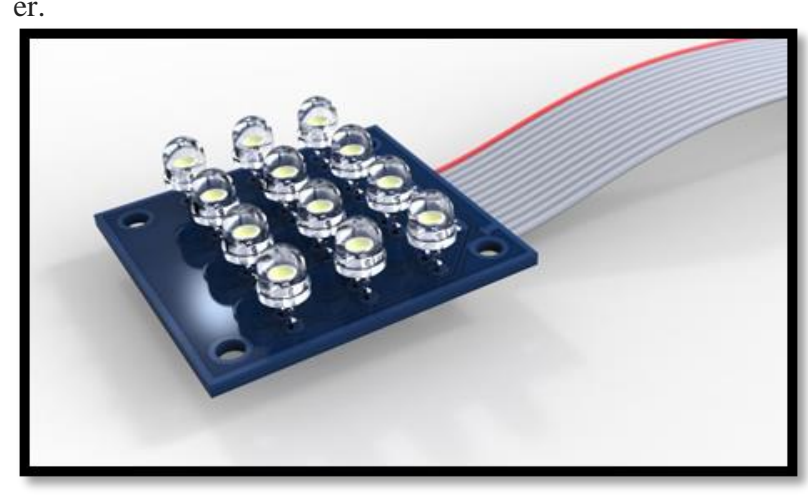

Fig 1.4: Led Array

\section{Objectives}

It used to give as an alternative or improve upload-directly to present wi-fi technology. To reestablished high speed connection speedy (in case of disaster problem). Li-Fi is used because it is fast and optical version of Wi-Fi which is very cheap. And to establish connections between two vehicles moving on the road.by this technology we can avoid accidents which is the major problem in now a days. The main reason for occurrence of accidents is unable to judge the road and unable to estimate the speed of leading vehicle. By this technology the following vehicle can get the information of leading vehicle like speed at that particular time and when the leading vehicle applies breaks suddenly this may reduce the occurrence of the accidents

\section{Methodology}

Communication between Vehicle to Vehicle and Ranging System has been proposed by using Spread Spectrum Technique. In this, a vehicle (Vehicle-A) provides the information about distance between target vehicle (Vehicle-B) and vehicle A. However, in this system, only one target vehicle is used in this paper, we also design multi target Communication and Ranging for vehicle to vehicle data transmission System by Using Spread Spectrum Technique. In this system, at the same time vehicle A can communicate with other target vehicles. From computer simulations, when interference signals are existing then we confirmed that this system become effective [1]. Vehicular ad hoc network (VANETs) that 
means devices have not access point is used for real time application such as (VANETs) can communicate with roadside units(RSU). By this communication provides information about traffic, accurate position of vehicle. For this purpose, number of protocols should be used to get desired output.

\section{Basic System}

The propose plan of activity for our venture is to start on optical remote correspondence show that gives high information rates (in the scope of $\mathrm{MHz}$ to $\mathrm{GHz}$ ) and transmission separations is close around $1 \mathrm{~m}$. For information transmission starting with one gadget then onto the next gadget required LED. In this framework at the transmitter area input information provide for the exchanging control framework. In light of the information, the exchanging control creates a surge of 0 s along these lines encoding the information in double. The yield of this control is given to the variety of Led's which OFF and ON at to a great degree high speeds. This ON-OFF adjustment of the LED light transmits the information. Driven is the decision for light source since it expends less power when contrast with fluorescent light or a light. It devours less power that is one-tenth power prerequisite when contrasted with customary strategies for lightning. Additionally, the lifetime a common LED knob is a few countless hours. Led's are additionally quick exchanging with great perceivability. Accordingly, Led's are perfect for use as the downlink transmitter. For the uplink transmitters, Infrared (IR) can be been the uplink transmitter for client accommodation. This abstains from fitting a LED light source on or by the cell phones. The get segment comprises of a photograph diode, for example, Infrared germanium round and hollow identifier and silicon photograph locator. The photograph identifier removes the approaching got flag based the grouping of $0 \mathrm{~s}$. The demodulated flag is then sent to a channel obliterate undesirable clamor. This channel flag is then open up utilizing signal intensification system. The channel and open up flag is then given to a yield gadget, for example, a LCD show or speaker. The info flag is in this manner remotely transmitter and collector. In this manner Li-Fi arrange is built up. The usefulness of the building squares of the framework is depicted straightaway. The information source e.g. (speed sensor) peruses the speed of the vehicle. The speed information from the sensor is crest to top AC voltage so it will be changed over to DC voltage to be meaningful by the microcontroller. At that point the information will be prepared by microcontroller (e.g. to think about between the present and past speed). New handled information will then be transmitted to the LED driver. Driven driver will make the ebb and flow consistent to ensure LED. At that point, information will transmit by the LED light.

The information which is to be transmitted is inserted in the light pillar. It is encouraged to the light driver which drives a LED lamp. The collector dongle comprises of a photograph identifier, intensification and preparing unit and information converter. Here, the information is again changed over to electric flag which is then sustained to a PC or portable.

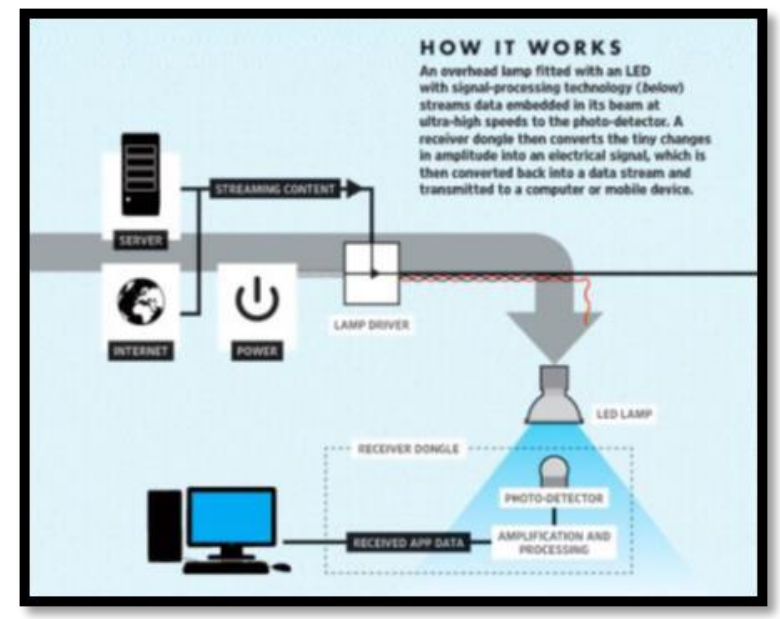

Fig 4 Basic Design

The above innovation can be utilized for street security and movement administration. One can make utilization of head lights and tail lights of vehicles. As each vehicle has relegated on number, one exceptional code is allocated to each vehicle. The head light and tail light transmit this code after a consistent interim. At the point when the vehicle goes through the toll court, the code is send to the PC at toll square. Finish data about your vehicle is then shown on the pc. Activity police can make utilization of this data if required. Individual at that point pays the toll (Road Service Tax) and push forward. In this manner data about every last vehicle passed, Number of vehicles go in one day and aggregate administration impose gathered is recorded in focal server of such street benefit charge framework. The total framework in this way winds up straightforward to a typical citizen. Every one of the PCs at all toll courts are arranged together. Accordingly, they can share the required data about every single vehicle. will make the flag green making Green Corridor for it.

\section{System Design}

The machine requires a transmitter and a receiver in every automobile in each rear and the front aspects of the automobile. For this reason, extra scenarios can be applicable.

- This system will detect the speed of the leading vehicle every time and transmit to the following vehicle

- This will be possible by toggling the led's for every particular speed within a range of $10 \mathrm{nsec}$

- And when the vehicle stops suddenly it should notify the following vehicle.

\subsection{Scenario}

The main scenario is when a vehicle stops suddenly while moving the vehicle which is following that vehicle should be notified which makes the driver to be alert and may avoid collision. In this every event is assigned with a sequence and if that event occurs then that sequence will be transmitted to the receiver using the led array. The events may be speed of leading vehicle, brakes are applied or there is any sudden change in the speed of the vehicle and this method is also used to regulate the traffic by replacing the street lights with led's if the is any road block or any accident happened in that road the sensors will transmit that particular stream of data to all the cars which are in that route and helps to avoid that route and this information may also useful for the police and ambulance to reach the spot when ever happened. 


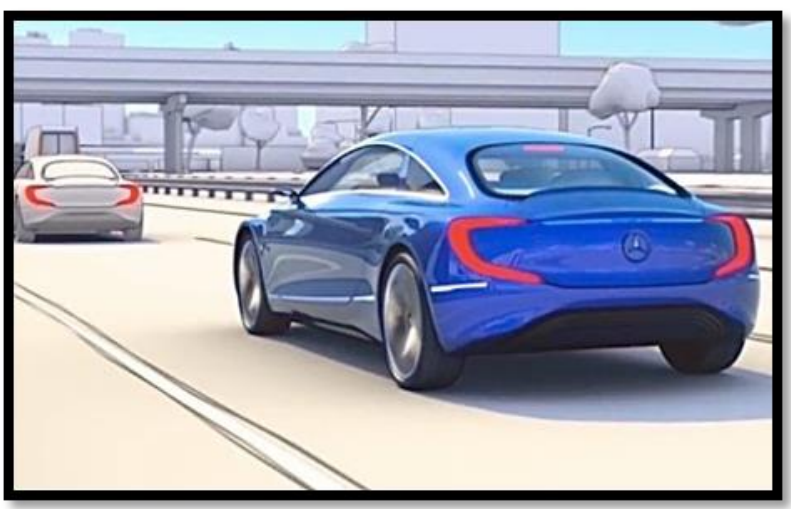

Fig 5.1 First scenario of vehicle to vehicle communication using Li-Fi

The led are made to toggle with a Nano seconds range to generate a sequence of data, the led's are made to toggle using an Arduino and when a particular event occurred then Arduino will make the led's to toggle according to the event, the event will assign to a particular toggle range of Nano seconds and when that events occurred then the data will be sent to the receiver and the receiver will be a photodiode when the light from the led fall on the photodiode it will generate an electrical pulse for every event and it will displays the message for that particular event and makes a sound.

\section{Conclusion}

The idea of Li-Fi will present alongside existing strategies and traditional patterns utilized for vehicle to vehicle correspondences. In this undertaking plans to propose a financially savvy answer for lessen mischances in Oman, the outline rules. The equipment angles with respect to the advancement of a VLC correspondence framework comprising of a business LED-based movement light and a vehicle will mount beneficiary. We will exhibit the approach we take after; a portion of the challenges we experience and clarify the decisions we have made. All through the usage procedure, we additionally endeavor on keeping the execution cost as low as would be prudent. Because of inaccessibility of all framework segments, sending information through Li-Fi little scale model.

\section{References}

[1] 1.Kiyoshi MIZUI, Masao NAKAGAWA, "Vehicle-to-Vehicle Multi Target Communication and Ranging System Using Spread Spectrum Technique".

[2] 2. "The phototransistor". Bell Laboratories RECORD. May 1950

[3] 3.Jump up^ Held. G, Introduction to Light Emitting Diode Technology and Applications, CRC Press, (Worldwide, 2008).

[4] 4.Harald Haas. "Harald Haas: Wireless data from every light bulb". ted.com.

[5] 5.Jump up^ Tsonev, Dobroslav; Videv, Stefan; Haas, Harald (December 18, 2013). "Light fidelity (Li-Fi): towards all-optical networking". Proc. SPIE. Broadband Access Communication Technologies VIII. 9007 (2)

[6] 6.M. N. N. A. Asghar, M.H, "Principle application and vision in the internet of things (iot)," in Communication Technologies (GCCT), 2015 Global Conference.

[7] R.C. Y. O. K. Vithanage, C, "A comparison of the popula home automation technologies,"

[8] R. Pavithra, D., "Iot based monitoring and control system for home automation,"

[9] T. Ming Zhao, Chua, "Automatic face and gesture recognition, 2008. Fig '08. 8th IEEE international conference on,"

[10] S. Cuomo, P. D. Michele, A. Galletti and F. Piccialli, "A Cultural Heritage Case Study of Visitor Experiences Shared on a Socia Network,"

[11] 2015 10th International Conference on P2P, Parallel, Grid, Cloud and Internet Computing (3PGCIC), Krakow, 2015, pp. 539-544.
[12] Angelo Chianese, Fiammetta Marulli, Francesco Piccialli, Paolo Benedusi, Jai E. Jung, An associative engines based approach supporting

[13] collaborative analytics in the Internet of cultural things, Future Generation Computer Systems, 2016.

[14] Wu X, Kumar V, Ross Quinlan J, Ghosh J, Yang Q, Motoda H et al. Top 10 algorithms in data mining. Knowledge and Information Systems.2007;14(1):1-37. 